\title{
Radiation-Enhancing Reflector
}

\author{
C. A. Valagiannopoulos ${ }^{1}$ and S. A. Tretyakov ${ }^{2}$ \\ ${ }^{1}$ Department of Physics, School of Science and Technology \\ Nazarbayev University, 53 Qabanbay Batyr Ave., Astana, KZ-010000, Kazakhstan \\ 2 Department of Radio Science and Engineering, School of Electrical Engineering \\ Aalto University, P.O. Box 13000, FI-00076, Finland
}

\begin{abstract}
We show that it is possible to realize a passive planar reflector whose reflectivity (close to the resonant frequency of the reflector structure) is larger than unity. This reflector is strongly coupled to evanescent fields of external sources and most effectively extracts power from them. Next, this power can be launched into far zone if the surface of the reflector is perturbed by some small passive scatterers. Used as electrically large reflector antennas, such planar surfaces show superdirectivity properties (the effective area is larger than the geometrical area of the reflector) while there is no need for careful subwavelength control over the amplitude and phase of the surface currents.
\end{abstract}

Index Terms-conjugate-matched layer, reflector, superdirectivity.

\section{INTRODUCTION}

If a small antenna (an electric dipole, for example) is positioned close to a planar passive mirror, the radiated field in the far zone is the sum of the primary field created by the source and the field reflected from the mirror. In the limit of zero losses in the reflector, the amplitude of the total field in a certain direction can vary from zero (when the reflected field totally cancels the primary field) to the double value of the primary field strength, when the two fields interfere constructively.

In this work, we show that it is possible to realize planar resonant reflectors which are able to enhance far-zone radiated fields more than two times. These reflectors not only reflect the incident power, but they are able to extract additional power from the primary source and launch it into the far zone. Although the proposed reflector is overall passive, it appears that the reflected power is considerably larger than the incident power radiated by the primary source into free space.

\section{THEORY AND NUMERICAL RESUlTS}

The main idea of the proposed approach is to create an artificial surface which could maintain high-quality resonant surface modes. Bringing a small source close to the surface, we can excite high-amplitude fields oscillating in the vicinity of the artificial mirror. Next, we want this surface to operate as an antenna, coupling its resonant modes to free space. Obviously, surface modes localized at an infinite homogeneous plane do not couple to the propagating plane waves, and the resonant oscillating surface currents do not radiate. To allow far-field radiation, we perturb the surface adding a set of small scatterers in the near vicinity of the surface. In this way: (i) we enhance the near fields of a small primary antenna, effectively extracting power from the source, and (ii) we launch this power into far zone.

As suitable resonant surfaces we propose to use the recently reported [1] slabs of uniaxial materials with the relative material parameters (transverse relative permittivity $\varepsilon_{r t}$, transverse relative permeability $\mu_{r t}$ and normal relative permittivity $\varepsilon_{r n}$ ) satisfying the relations

$$
\varepsilon_{r t}=\mu_{r t}=\frac{1}{\varepsilon_{r n}}=a-j b,
$$

with a negative value of $a<0$ and lossy transverse components: $b>0$. Here we consider only TM polarized waves since the relations for the TE polarization can be obtained using the duality principle. It is easy to show that the wave impedance of plane waves in such a medium is equal to the complex conjugate of the free-space wave impedance, for all propagating or evanescent plane waves [1]. To ensure that the medium is overall passive for all modes, we add an extra loss term $\delta>0$ to the normal component of the permittivity: $\varepsilon_{r n}=\frac{1}{\varepsilon_{r t}}-j \delta=\frac{1}{a-j b}-j \delta$.

We position an electrically thin layer (thickness $L$ ) of such material at a PEC ground plane, as shown in Fig. 1(a). We excite the structure by an electric dipole line source positioned at distance $g$ from the boundary material-air and tilted by the angle $\theta$ in the general case, as in [3]. The propagating plane waves created by the primary source penetrate into the slab without reflection (because for all propagating modes the wave impedances of the slab medium and of vacuum are equal) and get reflected from the PEC ground plane back into free space. Assuming that the losses are small (parameter $b$ is negligible), nearly all power carried by propagating waves is reflected as from a PEC mirror.

On the other hand, the impedance of evanescent plane waves created by the same source is imaginary, and at the input surface these waves see the complex-conjugate value of that. Under this resonant condition, the source excites very strong fields localized at the input surface of the slab. In the twodimensional configuration (excitation by an infinite dipole line along the $z$-axis as shown in Fig. 1), the spectral density of the secondary field created by the slab and the PEC mirror can be analytically solved. The spectral density is defined as follows:

$$
H_{\mathrm{sec}}(x, y)=\int_{-\infty}^{+\infty} S_{\mathrm{sec}}\left(k_{t}\right) e^{\kappa_{0}\left(k_{t}\right) x-j k_{t} y} d k_{t},
$$




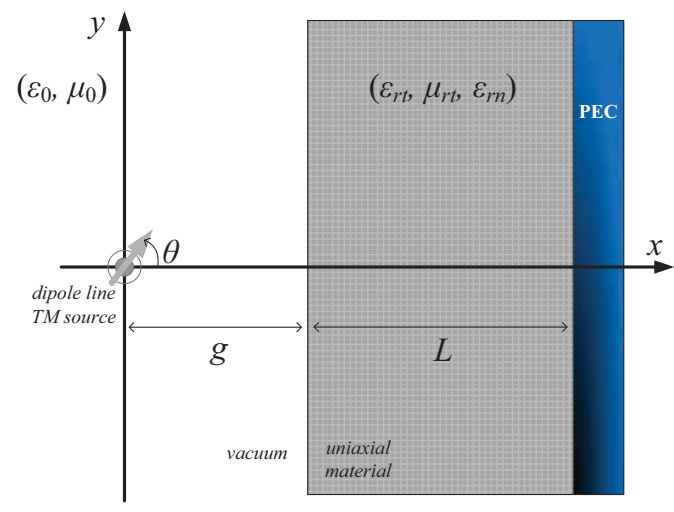

(a)

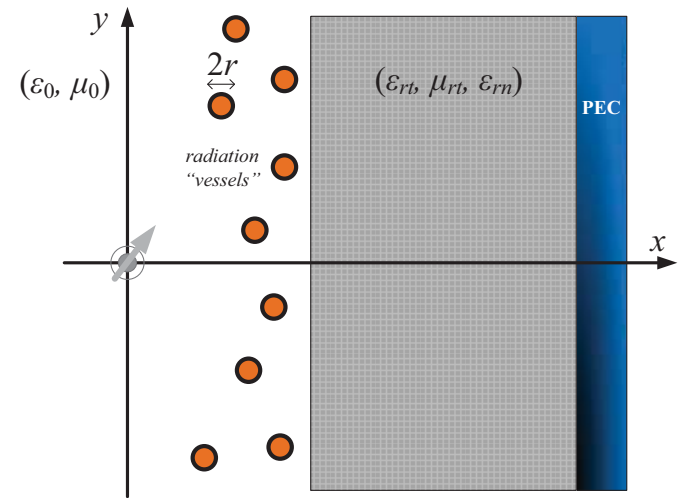

(b)

Fig. 1. (a) The test-bed configuration of a grounded electrically thick slab of uniaxial material $\left(\varepsilon_{r t}, \mu_{r t}, \varepsilon_{r n}\right)$ with thickness $L$ is excited by a TM electric dipole line inclined by angle $\theta$ located at a small distance $g$ from the air-medium interface. (b) The same structure in the presence of a cluster of electrically thin (radius $r$ ) circular cylinders positioned in the near field.

where $k_{t}$ is the transverse wavenumber. The normal component of the propagation constant $\kappa_{0}\left(k_{t}\right)=\sqrt{k_{t}^{2}-k_{0}^{2}}\left(k_{0}\right.$ is the free space wavenumber) is evaluated with a positive real part and in case it is zero, as a positive imaginary number. The results of example calculations are shown in Fig. 2, where the magnitude of the spectral spatial density function $S_{\mathrm{sec}}\left(k_{t}\right)$ determining the secondary magnetic field is represented as a function of $k_{t} / k_{0}$. The evaluated quantity is normalized by the constant value $S_{\text {inc }}\left(90^{\circ}\right)$ of the corresponding spectral density of the incident magnetic field when $\theta=90^{\circ}$. Here, we compare the case when the slab is a conventional uniaxial perfectly matched layer (PML), with a positive value of $a$, and the proposed conjugate-matched layer (CML) with a negative value of $a$. In the first case, the evanescent waves $\left(\left|k_{t}\right|>k_{0}\right)$ very quickly decay with increasing tangential wave numbers, as usually (Fig. 2(a)). In the second case which we study here, the propagating waves have the same amplitudes as for the PML slab (simple reflections in a PEC plane), but the evanescent fields are resonantly enhanced by several orders of magnitude (Fig. 2(b)).

However, these high-amplitude fields are evanescent and do

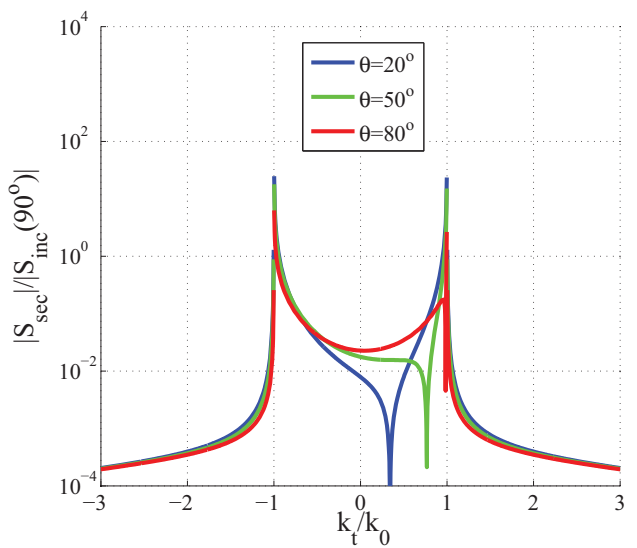

(a)

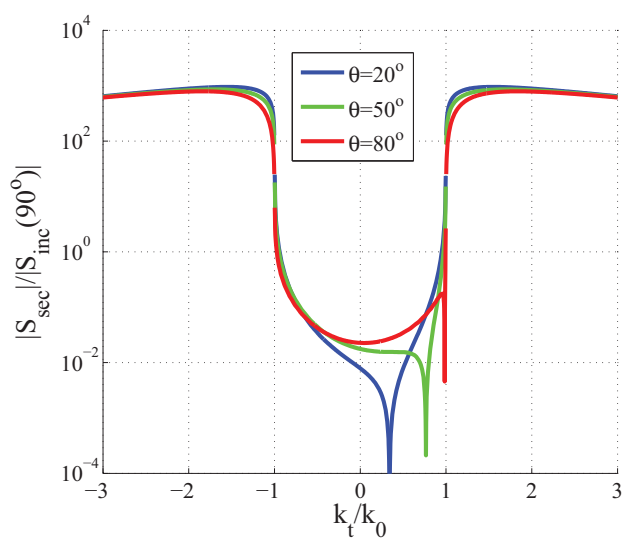

(b)

Fig. 2. The magnitude of the spatial spectral density function of the secondary field $S_{\mathrm{sec}}\left(k_{t}\right)$ at $x=0^{-}$with respect to normalized wavenumber $k_{t} / k_{0}$ for various inclination angles $\theta$. (a) $a=2$ (PML) and (b) $a=-2$ (CML). Common plot parameters: $b=0.1, \delta=0.001, g=0.03 \lambda_{0}, L=3 \lambda_{0}$. The represented quantity is normalized by the integrand of the incident field when the dipole line is vertical $\left(\theta=90^{\circ}\right)$.

not couple to propagating modes of free space. To launch their energy into far zone, we introduce an array of small scatterers in the vicinity of the resonant surface as shown in Fig. 1(b). They can be placed randomly in the near field of the slab were huge field (and power) concentration is developed due to the maximal evanescent waves excitation. For simplicity of calculations, we consider an array of $N$ thin perfect magnetic conductor (PMC) cylinders positioned at distance $w$ from the interface CML-air. The PMC pins are modeled by the boundary condition $H_{z}=0$ and their effect is analytically obtained through integral equation formulation described in [4].

We can apply the stationary phase method to evaluate the incident $H_{\text {inc }}(\rho, \varphi)$, secondary $H_{\text {sec }}(\rho, \varphi)$ and total $H(\rho, \varphi)$ magnetic field components in the far zone $\left(k_{0} \rho \rightarrow+\infty\right)$. In this way, we can analytically obtain the corresponding azimuthal profiles $h_{\mathrm{inc}}(\varphi), h_{\mathrm{sec}}(\varphi)$ and $h(\varphi)$. 


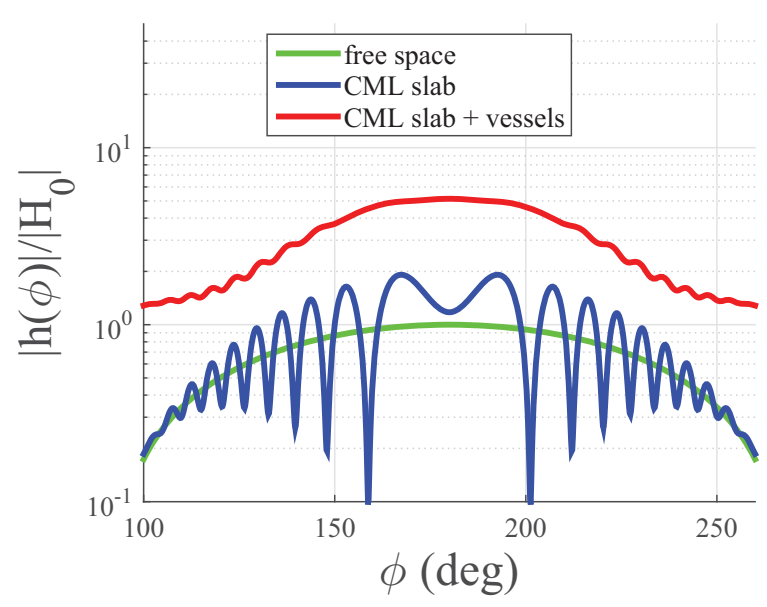

Fig. 3. The (normalized by the amplitude of the field existing without reflector $H_{0}$ ) azimuthal profiles of the magnetic fields when the source is alone in free space $h_{\text {inc }}(\varphi)$, when it excites the grounded CML slab $h_{\text {inc }}(\varphi)+h_{\text {sec }}(\varphi)$, and when the cylindrical radiation vessels are used $h(\varphi)$. Plot parameters $a=-2, b=0, \delta=0.01, g=0.1 \lambda_{0}, w=0.05 \lambda_{0}$, $r=0.01 \lambda_{0}$.

In Fig. 3 we present the magnitudes of these functions normalized by $H_{0}$ which is the value of the incident field at $\varphi=180^{\circ}$ (at broadside). Three cases are considered. The green curve shows the far-field pattern of the primary dipoleline source radiating into infinite free space. This is, obviously, just the usual cosine pattern of a dipole, with the maximum amplitude set to unity. The blue line shows the pattern of the same dipole line source in the vicinity of the conjugatematched layer without perturbing cylinders (configuration of Fig. 1(a)). As expected, in some directions the reflected propagating waves constructively interfere with the primary field of the source, and the amplitude is nearly doubled (the losses in the slab are small). Although the evanescent fields in the vicinity of the source are very strong, they do not contribute to far-field radiation. Finally, the red curve shows the result in the presence of perturbing pins, which we call radiation vessels. Clearly, the fields radiated into far zone are in this case dramatically enhanced at all angles.

\section{DisCUSSION AND CONCLUSION}

The proposed radiation-enhancing reflectors consist of a uniaxial slab of a double-negative material (the real parts of the permittivity and permeability are negative). In the literature, many design solutions for double-negative metamaterials can be found, which can be possibly used to realize such supermirrors in practice. Although in the examples shown in this abstract the overall response of the material is always passive for all plane-wave modes inside the layer, the normal components of the material tensors have positive real parts, corresponding to gain. This property makes potential realizations more difficult than for conventional double-negative materials. However, the results of paper [1] show that this difficulty can get overcome, since similar electromagnetic properties can be achieved even for lossless normal components, if the values of the tangential components are chosen properly.

Although the proposed reflector is planar and its area is electrically large, its effective area is larger than the geometrical one. Basically, we obtain a surface which (at its resonant frequency) is "more bright than the ideal PEC mirror", and this property holds even in the limit of the infinite planar reflector. In earlier work [1], [2] physically similar results were obtained for absorbers, which could be made "more black than the ideal black body" in its classical definition. However, the earlier results hold only for bodies of a finite size, as the necessary coupling of locally oscillating fields to far-field was made possible due to the surface curvature. In that scenario [2], increasing the body size leads to decreasing the surface curvature, that is, to weaker coupling of resonant evanescent modes to free space. Here we remove this limitation by perturbing the surface. Thus, the results of this paper can have implications also for realization of extreme absorbers as well as for engineering of thermal radiation.

\section{ACKNOWLEDGMENT}

The authors would like to thank Dr. S. Maslovski and Prof. C. Simovski for useful discussions.

\section{REFERENCES}

[1] C. A. Valagiannopoulos, J. Vehmas, C. R. Simovski, S. A. Tretyakov, and S. Maslovski, "Electromagnetic energy sink", Physical Review B, vol. 92, no. 245402, 2015.

[2] S. I. Maslovski, C. R. Simovski, S. A. Tretyakov, "Overcoming black body radiation limit in free space: metamaterial superemitter", New Journal of Physics, vol. 18, no. 013034, 2014.

[3] C. A. Valagiannopoulos, M. S. Mirmoosa, I. S. Nefedov, S. A. Tretyakov and C. R. Simovski, "Hyperbolic-metamaterial antennas for broadband enhancement of dipole emission to free space", Journal of Applied Physics, vol. 116, no. 163106, 2014.

[4] C. A. Valagiannopoulos, "On examining the influence of a thin dielectric strip posed across the diameter of a penetrable radiating cylinder", Progress In Electromagnetics Research C, vol. 3, pp. 203-214, 2008. 